\title{
Ideal planting space for merbau (Intsia bijuga) forest plantations in Papua based on distance-dependent competition
}

\author{
ELIESER VIKTOR SIRAMI ${ }^{1,2, \bullet}$, DJOKO MARSONO $^{1}$, RONGGO SADONO $^{1}$, MUHAMMAD ALI IMRON $^{1}$ \\ ${ }^{1}$ Faculty of Forestry, Universitas Gadjah Mada. Jl. Agro No. 1, Bulaksumur, Sleman 55281, Yogyakarta, Indonesia \\ ${ }^{2}$ Faculty of Forestry, Universitas Papua. Jl. Gunung Salju, Amban, Manokwari 98314, West Papua, Indonesia. \\ Tel.: +62-986-211065, Fax.: +62- 986-211065, "email: siramieli@yahoo.co.id
}

Manuscript received: 30 September 2018. Revision accepted: 9 November 2018.

\begin{abstract}
Sirami EV, Marsono D, Sadono R, Imron MA. 2018. Ideal planting space for merbau (Intsia bijuga) forest plantations in Papua based on distance-dependent competition. Biodiversitas 19: 2219-2231. Distance-based competition between merbau (Intsia bijuga (Colebr.) Kuntze) and neighboring trees could be applied to determine the planting space between merbau trees and shade trees in plantations. This research was conducted to identify the characteristics of merbau competition with neighboring trees and determine the ideal spacing of merbau trees. The sampling design using was the systematic line technique with hypothetical plot and sample tree as the quadrant center. The competitors were determined using a virtual high approach and header contact. Distance-based competitions were identified using the Hegyi index. Sixteen species had the highest competition index because of their dominance in the tree structure, namely Pometia coriacea, Intsia bijuga, Pimelodendron amboinicum, Horsfieldia laevigata, Palaquium amboinense, Pometia pinnata, Garcinia sp., Spathiostemon javensis, Prunus costata, Sterculia macrophylla, Terminalia complanata, Lepiniopsis ternatensis, Horsfieldia irya, Dysoxylum octandrum, Buchanania arborescens, and Ficus similis. Merbau responded to the high intensity of competition by tilting its canopy in the opposite direction to the position of the competitors' canopy, making an irregular canopy shape, and growing the first branch at the lowest point on the stem. The ideal planting space for merbau trees in the plantations was 3-7 m.
\end{abstract}

Keywords: Distance-dependent competition, Gunung Meja, merbau plantations, planting space, West Papua

\section{INTRODUCTION}

Currently, merbau (Intsia bijuga (Colebr.) Kuntze) population faces serious threats due to timber production (Newman and Lawson 2005; Marler 2015) and its natural habitat destruction in the lowland rainforest of New Guinea (Vincent et al. 2015; Margono et al. 2014). A plantation forest with native tree species is an alternative to prevent these threats (Barua et al. 2014; Jacovelli 2014; Bremer and Farley 2010). However, the cultivation of native trees requires the availability of relevant ecological information, one of which is the characteristic of merbau competition with surrounding trees.

In the Papua forest, merbau trees naturally grow in hight dense vegetation conditions due to the density of plants. The density of plants in tropical forests makes distances between trees get closer. It affects the survival of the trees (Zhu et al. 2015; Fraver et al. 2014), because the close distance between trees increases the effect of competition (Maleki et al. 2015; Contreras et al. 2011; Tome and Burkhart 1989). Competition affects the diameter growth, height, width, and shape of tree canopy (Kunstler et al. 2011; Potvin and Dutilleul 2009; Thorpe et al. 2010). Therefore, competition is an essential factor driving forest dynamics (Sanchez-Salguero et al. 2015; Coomes and Allen 2007; Kunstler et al. 2011).

Several facts regarding competition between trees which become important references in silviculture of plantation forests, such as determining planting space, have been explained in previous studies. Competition is one of the plant structuring processes that led to the formation of a variety of stand structures (Craine and Dybzinski 2013; Amiri and Naghdi 2016). Differences of the nature of individuals or species of the tree have a strong influence on the competition (Bennett et al. 2016). If competition is based on niche differentiation, then same-species competition is stronger than different species competition (MacArthur and Levins 1967). However, in natural forests, same-species competition can occur altogether with different species competition. Therefore, each tree species will provide different intensity and effect of competition depending on the ability to compete with others. The ability of competition is a function of growth space, activity, and distribution of space and time of each plant to get resources depending on the combination of plant characteristics (Grime 1979). Furthermore, the intensity of competition depends on the spatial relationship between plants and their neighbors, the impact of the availability of resources on the two competing trees, and the ability of the plant to compensate for the effects of competition through architectural and physiological plasticity (Grace 1995).

Spatial relationship between trees is one of the most important factors in competition. Therefore, a distancebased competition study is very important to be carried out as a reference to formulate the ideal spacing of merbau tree for its cultivation in plantations. Distance-based competition, when associated with morphological character of the stand, produces certain distance with the certain intensity of competition. The higher size of the trees and the closer the space between trees, the more intense the 
competition (Tome and Burkhart 1989). Furthermore, the distance with the highest intensity of competition can be used as a reference in the formulation of planting space between merbau trees and shade trees, because merbau its regeneration is semi-tolerant.

Planting space is a fundamental silvicultural factor in plantations; because it affects the level of harvest, wood rigidity and strength, and tree regeneration (Skovsgaard and Vanclay 2008; Clark III et al. 2008; Sansevero et al. 2011). According to Turner (2004), semi-tolerant species need medium shade to grow appropriately. Therefore, concerning the growth of merbau, the ideal spacing needs to be determined because it is closely related to the shadow of neighboring trees which can inhibit optimum light or cause light intensity to be too high for merbau in the dry season.Prior to this study, there has been no research on competition between merbau and neighboring tree species in Papua lowland rainforest and also the ideal spacing for merbau trees domesticated in plantations. This study aimed to identify the characteristics of the distance-dependent competition of merbau with neighboring trees and formulate the ideal spacing for merbau trees in plantations.

\section{MATERIALS AND METHODS}

\section{Study area}

This research was conducted at Gunung Meja Nature Tourism Park of Manokwari (GMNTPM), West Papua, at $134^{\circ} 03^{\prime} 17^{\prime \prime}-134^{\circ} 04^{\prime} 05^{\prime \prime} \mathrm{W}$ and $0^{\circ} 51^{\prime} 29-0^{\circ} 52^{\prime} 59^{\prime \prime} \mathrm{S}$ (Fig.1), in 2016 for 9 months. GMNTPM is one of the protected areas and plays a very important role for forest ecology. Apart from being a natural habitat for merbau, GMNTPM is a rough forest prototype for the Papua region. Merbau habitat at GMNTPM has an area of \pm 264 ha of rough soil surface (Sadono et al. 2014), from 460.25 ha in the entire area. The temperature under the forest canopy in the dry season is around $29-31^{\circ} \mathrm{C}$, rainfall for the last 13 years ranges from 1429 to $3419 \mathrm{~mm}$, while the sunshine ranges from 444 to $745 \mathrm{~h}$ (Statistics Agency of Manokwari Regency 2016). The soil texture is sandy clay soil with a soil surface depth of less than $50 \mathrm{~cm}$. Forest canopy ranges from 40 to $98 \%$, with slopes of $2-40 \%$, an altitude of 70-170 $\mathrm{m}$ above sea level (Sadono et al. 2014).

\section{Research procedure}

The initial survey was conducted using merbau distribution maps at GMNTPM. Data collection was performed using systematic line technique with hypothetical plot and sample trees as the quadrant center. This technique is the modification of several existing vegetation analysis techniques for the need of distance-based competition research in Papua lowland forests that have high tree density.

Observation lines were made only as a tracking guide. It was systematic and the distance between lines was 20 $\mathrm{m}$. When a merbau tree with a diameter of 10-19 cm was found, a quadrant observation plot was made by placing one sample tree as the center of the quadrant. Competitors were determined using the virtual canopy and high intersection approaches (Burkhart and Tome 2012). Trees that were designated as competitors were the closest neighboring trees in each quadrant which canopies were directly contacted the merbau canopy and higher than the merbau tree; or the closest trees which canopies were in the virtual height area of the merbau tree, even though the canopies did not intersect with the merbau tree's canopy, because it will be the last tree that blocks the light (Fig. 2).

The parameters of subject trees and competitors included species name, projection length of canopy diameter twice with the opposite direction, DBH, total height, clear bole height, canopy shape, and canopy direction.

\section{Data analysis}

The intensity of the competition was determined using the Hegyi's Competition Index (Contreras et al. 2011; Tome and Burkhart 1989). This index based on the hypothesis that the competitive effects of neighboring trees increase by the increasing size and closeness (Tome and Burkhart 1989).

$$
\mathrm{IK}_{\mathrm{mi}}=\sum_{\mathrm{j}=1}^{\mathrm{m}}\left(\frac{\mathrm{DBH}_{\mathrm{i}}}{\mathrm{DBH}_{\mathrm{m}}} \times \frac{1}{\mathrm{Dis}_{\mathrm{im}}}\right)
$$

The canopy area was determined using the canopy closure approach (Mueller-Dombois and Ellenberg 1974).

Canopy cover $\left(\mathrm{m}^{2}\right)=0,25 x\left(D t_{1}+D t_{2}\right)^{2}$

$\mathrm{IK}_{\mathrm{m}, \mathrm{i}}=$ intensity of competition between subject tree and competitors

$\mathrm{DBH}_{\mathrm{m}}=$ Diameter at breast height of the subject tree (cm)

$\mathrm{DBH}_{\mathrm{i}}=$ Diameter at breast height of competitors $(\mathrm{cm})$

Dis $_{\text {im }}=$ Distance between the subject tree and its competitors (m)

$\mathrm{n}=$ Number of competitors

$\mathrm{Dt}_{1}=$ Canopy diameter from the first measurement

$\mathrm{Dt}_{2}=$ Canopy diameter from the second measurement

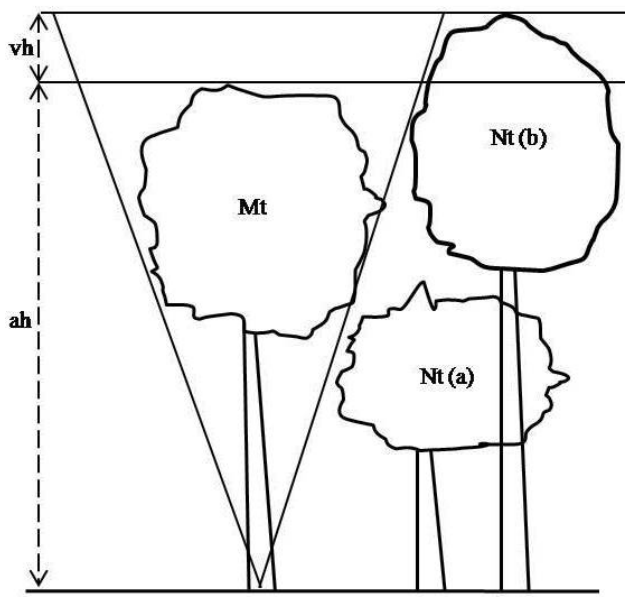

Figure 2. Determination of the competitor trees. Vh: virtual height, ah: actual height, Mt: merbau tree, $\mathrm{Nt}$ (a): neighbor tree a, $\mathrm{Nt}$ (b): neighbor tree $\mathrm{b}$. (neighboring tree $\mathrm{b}$ is chosen as a competitor tree) 


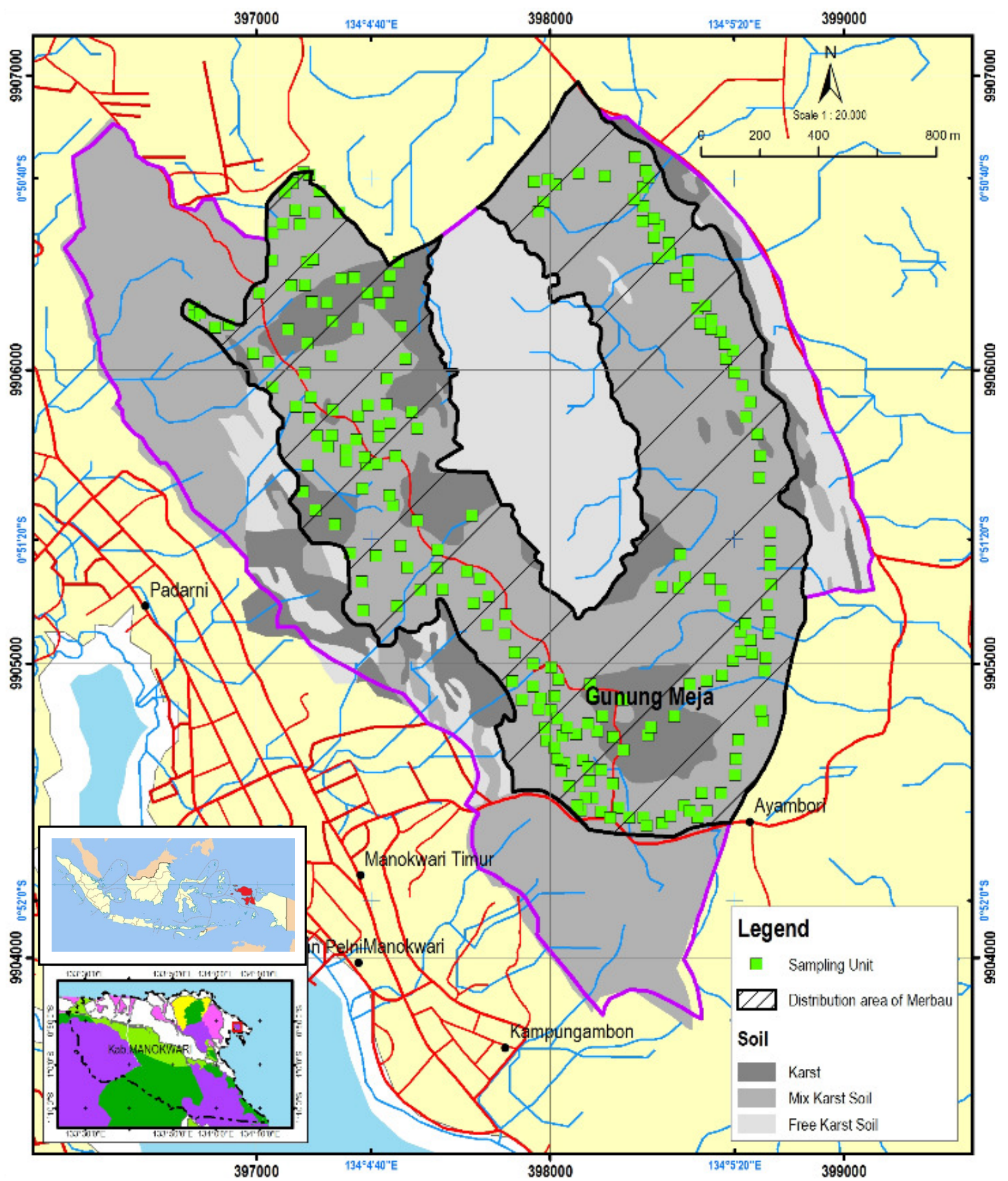

Figure 1. Reseach site in Gunung Meja Nature Tourism Park of Manokwari (GMNTPM), West Papua Province, Indonesia

\section{RESULTS AND DISCUSSION}

\section{Structure, the composition of neighboring tree species and the intensity level of competition}

Based on observation in 218 sampling units, 849 stands of merbau competitors were identified, consisting of 80 species, 58 genera, and 34 families. All competitors are native New Guinea species that had adapted to the rough land in GMNTPM.There are 2-3 competitors with 2-3 species within a range of 7 to $35 \mathrm{~m}$ per sampling unit. Competitors with diameter $>20 \mathrm{~cm}$ occupy strata A and B with canopy area between $2-4 \mathrm{~m}^{2}$ per tree, while trees with a diameter of $<20 \mathrm{~cm}$ occupy strata $\mathrm{C}$. Merbau stands which become target trees have a diameter of 11-17 $\mathrm{cm}$ in general. The total height ranges from 9 to $15 \mathrm{~m}$ and the canopy width varies between 1 and $3 \mathrm{~m}^{2}$ per tree. In addition, the number of merbau populations as the target tree and competitor trees is 328 stands. 
From 80 species of competitors, only 16 species have higher total Hegyis index scores, compared to the other 64 species (Table 1). The species with high intensity of competition are dominant trees. There were two large groups of trees dominated the forest structure when they reached the adult phase in GNMTPM. Species such as Spathiostemon javensis, Mallotus spp., Lepiniopsis ternatensis, Prunus costata, reached the adult phase in the $<20 \mathrm{~cm}$ diameter class, therefore it was very dominant. Species such as Pometia spp., Intsia spp., Pimelodendron amboinicum, Palaquium amboinense, several species of Myristicaceae, Burseraceae, and Meliaceae were very dominant in the class of $\geq 20 \mathrm{~cm}$ of diameter. However, in the class of $\geq 50 \mathrm{~cm}$, generally, there were only a few species such as Pometia coriacea, Palaquium amboinense, Intsia spp., sometimes also several stands of Geijera sp. and Dysoxylum spp. The long-standing tree adaptation in GNMTPM might cause this dominance.

The tree domination shows the ability to adapt to the rocky soil at GMNTPM and a better biological fitness level. The biological fitness level is indicated by a relatively good reproductive capacity such as the relatively large number of fruits and seeds, the anatomical structure and morphology of the seeds which strongly supports the mechanical, physical and biological germination and dispersion process. Competition is a form of coexistence among trees that starts from spatial distribution (Callaway 1995). Our findings show the dispersion process of dominant trees influences the level of presence around the merbau stand. Therefore, we assume that the spatial distribution and adaptability are very decisive with what species of merbau grows very close and competes with each other. The effect of distance will increase the intensity of competition if dominant tree species have faster growth rates than merbau. As well as, Pometia coriacea, Pimelodendron amboinicum, Prunus costata, Horsfieldia laevigata, Palaquium amboinense, Pometia pinnata, Spathiostemon javensis, Ficus similis, Lepiniopsis ternatensis, Horsfieldia irya, Dysoxylum octandrum, and Buchanania arborescens. The process of dispersing of these species is assisted by frugivorous animals of the bird and mammals group at GMNTPM, and also have faster growth rates than merbau.

\section{The most competitive trees species (MCTS)}

We termed the species with the highest intensity of competition as the most competitive trees species (MCTS). MCTS group with DBH $<30 \mathrm{~cm}$ has canopy area of 1.753$2.59 \mathrm{~m}^{2}$ in average, mean of Hegyi index of $0.20-0.60$ and distance of 3.65-5.69 $\mathrm{m}$. DBH group $\geq 30 \mathrm{~cm}$ has canopy area of $2.78-4.75 \mathrm{~m}^{2}$ in average, the distance of $5.30-6.60$ $\mathrm{m}$ and Hegyi index of $0.07-0.13$. The canopy area is directly proportional to $\mathrm{DBH}$ and distance, but it is inversely proportional to Hegyi index (Table 2). It means that the intensity of competition strongly influences the canopy area; the higher the intensity of competition is inhibited the canopy growth. Therefore, the canopy is used as the main reference in formulating the ideal spacing because it is closely related to light competition and growing space (Tremmel and Bazzaz 1993; Collins and
Wein 2000; Pretzsch 2014).

The competition also shows different outcomes when analyzed based on different species (Bennett et al. 2016), and growth rates. The merbau trees used as target trees were pre-mature trees (DBH 10-19 $\mathrm{cm}$ ). The goal was to obtain information on the effects of competition to predict how merbau reach a mature stage if cultivated in plantations. Therefore, we choose the most competitive tree species as a source of competitor data to form the ideal spacing.

Not only has the highest competition intensity, MCTS is also the most dominant species among competitors based on stand structure and dendrometric characteristics. The MCTS has a relatively closer average distance to merbau. The diameter sizes of the merbau and the competitor tree at each sample point are relatively large. It has a relatively wide canopy cover, a higher frequency of presence around merbau, higher total height average and the higher number of individuals than the other species. Therefore, MCTS requires greater growth space, thus competition with merbau stands is more intense in certain growth phases.

Table 3 shows the significant negative relationship of Hegyi index, total height and canopy area of MCTS $(p<$ 0.01 ). If there is a competition with high intensity, there is a tendency to inhibit the growth rate of the height and canopy area of both merbau and competitors.

Vertical growth barriers and tree canopy area are mechanical indicators that space is limited (Table 3). Large trees need more space to grow and they are superior to small plants in competition for light (Aarssen et al. 2014; Grace 1990; Goldberg 1996). MCTS indeed inhibits the absorption of light of merbau and the smaller surrounding trees and controls larger growing space. The number of MCTS stands is $77 \%$ of the total individual competitors of merbau, this is an indication of biological fitness that is beneficial to support its survival, including the ability to compete.

\section{The position of competitor trees}

The position of the tree was not the main factor of competition related to the light orientation, but it was closely related to the space needed for each stand to increase volume of the tree (Table 4). Competitors grew more in southeast direction, while the highest number of species was in the north position. Distance, DBH, canopy area, and total height generally indicated the same number for each position.

Competition for nutrition among trees strongly influences the rate of growth. The use of volume crown as a metrical measurement indicates that most strongly, the competition is triggered by the tree layout that intensifies the competition (Fraver et al. 2014). The tree planting layout and the intensity of competition affect the growth rate of the plants. Therefore, competition is seen as a fundamental ecological process that plays a major role in population dynamicity, plant survival, growth, and species replacement or succession is a fundamental ecological process that plays a major role in population dynamics, survival, growth and species replacement or succession (Peet and Christensen 1987; Maleki et al. 2015). 
Table 1. Structure, species composition, and Hegyi index of competitor trees

\begin{tabular}{|c|c|c|c|c|c|c|c|c|c|c|}
\hline Species & Family & $\begin{array}{l}\text { Hegyi } \\
\text { index }\end{array}$ & $\begin{array}{l}\text { Intensity of } \\
\text { competition }\end{array}$ & $\begin{array}{l}\text { Distance } \\
\text { (m) } \\
\text { mean } \pm \text { SD }\end{array}$ & $\begin{array}{l}\mathrm{DBH}(\mathrm{cm}) \\
\operatorname{mean} \pm \mathrm{SD}\end{array}$ & $\begin{array}{l}\text { Height }(\mathrm{m}) \\
\operatorname{mean} \pm \mathrm{SD}\end{array}$ & $\begin{array}{l}\text { Canopy } \\
\text { cover } \\
\left(\mathbf{m}^{2}\right)\end{array}$ & $\begin{array}{l}\text { Basal } \\
\text { area } \\
\left(\mathbf{m}^{2}\right)\end{array}$ & $\begin{array}{l}\Sigma \\
\text { indiv. }\end{array}$ & Freq. \\
\hline Aceratium opositifolium DC. & Elaeocarpaceae & 0.07 & Low & 6 & 26 & 10 & 3.25 & 0.053 & 1 & 0.005 \\
\hline Aglaia odorata Lour. & Meliaceae & 0.25 & Low & $14 \pm 1.74$ & $46.80 \pm 7.20$ & $15.62 \pm 11.38$ & 3.75 & 0.145 & 2 & 0.009 \\
\hline Aglaia spectabilis (Miq.) S.S. Jain \& S.Bennet & Meliaceae & 0.20 & Low & 4 & 23 & 18 & 1.75 & 0.042 & 1 & 0.005 \\
\hline Alstonia macrophylla W. ex G.Don & Apocynaceae & 1.09 & Low & $7 \pm 1.98$ & $44.12 \pm 19.21$ & $19.60 \pm 11.73$ & 15.25 & 0.533 & 6 & 0.028 \\
\hline Alstonia scholaris (L.) R.Br. & Apocynaceae & 0.14 & Low & 4 & 20 & 10 & 1.25 & 0.031 & 1 & 0.005 \\
\hline Antiaris toxicaria Lesch. & Moraceae & 0.91 & Low & $8 \pm 1.73$ & $34.92 \pm 25.74$ & $17.02 \pm 13.98$ & 13.50 & 0.442 & 6 & 0.028 \\
\hline Archidendron parviflorum Pulle & Leguminosae & 0.07 & Low & 5 & 30 & 18 & 3.00 & 0.071 & 1 & 0.005 \\
\hline Buchanania arborescens (Blume) Blume & Anacardiaceae & 2.70 & Medium & $15 \pm 1.87$ & $43.79 \pm 21.64$ & $18.01 \pm 13.71$ & 62.75 & 1.957 & 21 & 0.092 \\
\hline Callophyllum inophyllum L. & Clusiaceae & 0.81 & Low & $6 \pm 3.09$ & $46.47 \pm 24.53$ & $23.46 \pm 13.79$ & 24.50 & 0.858 & 8 & 0.032 \\
\hline Canarium indicum $\mathrm{L}$. & Burseraceae & 0.84 & Low & $7 \pm 0.58$ & $53.09 \pm 18.91$ & $28.48 \pm 14.02$ & 12.50 & 0.476 & 4 & 0.018 \\
\hline Carallia brachiata (Lour.) Merr. & Rhizophoraceae & 0.20 & Low & 3 & 27 & 19 & 2.00 & 0.057 & 1 & 0.005 \\
\hline Celtis latifolia (Blume) Planch. & Ulmaceae & 0.94 & Low & $9 \pm 2.38$ & $42.59 \pm 26.41$ & $25.54 \pm 16.46$ & 25.25 & 0.783 & 8 & 0.037 \\
\hline Cerbera floribunda K.Schum. & Apocynaceae & 0.83 & Low & 1 & 12 & 10 & 1.18 & 0.011 & 1 & 0.005 \\
\hline Chionanthus ramiflorus Roxb. & Oleaceae & 0.88 & Low & $7 \pm 1.82$ & $26.81 \pm 15.99$ & $14.69 \pm 9.71$ & 8.00 & 0.189 & 5 & 0.009 \\
\hline Cinnamomum sintoc Blume & Lauraceae & 0.15 & Low & 6 & 20 & 13 & 2.25 & 0.031 & 1 & 0.005 \\
\hline Citronella sp. & Cardiopteridaceae & 0.31 & Low & $6 \pm 1.98$ & $37.73 \pm 12.27$ & $14.41 \pm 11.59$ & 4.75 & 0.111 & 2 & 0.009 \\
\hline Cleistanthus myrianthus (Hassk.) Kurz & Phyllanthaceae & 0.45 & Low & $7 \pm 2.98$ & $13.62 \pm 9.38$ & $13.04 \pm 5.96$ & 2.00 & 0.021 & 2 & 0.009 \\
\hline Cryptocarya massoy (Oken) Kosterm. & Lauraceae & 0.17 & Low & $6 \pm 3.59$ & $27.41 \pm 24.59$ & $15.62 \pm 11.38$ & 4.50 & 0.106 & 2 & 0.009 \\
\hline Cryptocarya sp. & Lauraceae & 0.29 & Low & $4 \pm 4.00$ & $36.04 \pm 28.96$ & $21.66 \pm 10.34$ & 6.00 & 0.167 & 2 & 0.023 \\
\hline Crysophyllum sp. & Sapotaceae & 0.12 & Low & $14 \pm 4.55$ & $30.62 \pm 26.38$ & $21.24 \pm 12.76$ & 4.50 & 0.128 & 2 & 0.009 \\
\hline Dehaasia $\mathrm{sp}$ & Lauraceae & 0.22 & Low & $9 \pm 3.82$ & $56.30 \pm 25.04$ & $17.82 \pm 15.51$ & 7.25 & 0.428 & 3 & 0.014 \\
\hline Diospyros papuana Valeton ex Bakh. & Ebenaceae & 0.13 & Low & $7 \pm 4.59$ & $36.24 \pm 27.76$ & $16.21 \pm 14.79$ & 5.00 & 0.162 & 2 & 0.005 \\
\hline Drypetes acuminata P.I.Forst. & Putranjivaceae & 0.09 & Low & 7 & 28 & 17 & 3.25 & 0.062 & 1 & 0.009 \\
\hline Dysoxylum mollissimum Blume & Meliaceae & 0.30 & Low & $5 \pm 3.76$ & $35.75 \pm 19.58$ & $19.18 \pm 12.15$ & 6.50 & 0.191 & 3 & 0.014 \\
\hline Dysoxylum mollissimum subsp. molle (Miq.) Mabb. & Meliaceae & 0.25 & Low & $4 \pm 2.44$ & $68.39 \pm 44.95$ & $24.84 \pm 15.82$ & 16.75 & 0.778 & 3 & 0.014 \\
\hline Dysoxylum octandrum (Blanco) Merr. & Meliaceae & 2.72 & Medium & $8 \pm 1.59$ & $49.62 \pm 19.38$ & $23.36 \pm 16.27$ & 68.75 & 1.764 & 16 & 0.073 \\
\hline Elaeocarpus angustifolius Blume & Elaeocarpaceae & 1.16 & Low & $7 \pm 2.90$ & $39.19 \pm 14.06$ & $19.60 \pm 13.65$ & 22.75 & 0.532 & 8 & 0.037 \\
\hline Elaeocarpus serratus L. & Elaeocarpaceae & 0.13 & Low & $7 \pm 5.76$ & $59.20 \pm 48.80$ & $34.39 \pm 13.61$ & 11.50 & 0.691 & 3 & 0.014 \\
\hline Endiandra sp. & Lauraceae & 0.08 & Low & 6 & 28 & 14 & 1.00 & 0.062 & 1 & 0.005 \\
\hline Ficus benjamina L. & Moraceae & 0.36 & Low & $9 \pm 4.10$ & $119.18 \pm 53.68$ & $19.15 \pm 16.85$ & 34.25 & 4.610 & 7 & 0.032 \\
\hline Ficus similis Merr. & Moraceae & 2.62 & Medium & $8 \pm 3.83$ & $27.21 \pm 17.68$ & $21.21 \pm 8.32$ & 38.00 & 0.742 & 18 & 0.069 \\
\hline Ficus sp. & Moraceae & 0.71 & Low & $11 \pm 2.64$ & $88.06 \pm 28.44$ & $20.17 \pm 9.33$ & 37.75 & 2.619 & 8 & 0.037 \\
\hline Flacourtia inermis Roxb. & Salicaceae & 0.11 & Low & 4 & 32 & 15 & 3.75 & 0.080 & 1 & 0.005 \\
\hline Garcinia picrorhiza Miq. & Clusiaceae & 1.19 & Low & $9 \pm 3.65$ & $33.46 \pm 22.21$ & $17.85 \pm 14.65$ & 27.75 & 0.757 & 12 & 0.147 \\
\hline Garcinia sp. & Clusiaceae & 6.65 & Medium & 6.65 & $41.20 \pm 17.34$ & $20.39 \pm 12.07$ & 96.25 & 3.204 & 41 & 0.032 \\
\hline Gnetum genemon L. & Gnetaceae & 0.41 & Low & 2 & 11 & 12 & 0.50 & 0.009 & 1 & 0.005 \\
\hline Gymnacranthera farquhariana (Hook.f. \& Thomson) Warb. & Myristicaceae & 0.36 & Low & 1 & 20 & 10 & 2.75 & 0.031 & 1 & 0.005 \\
\hline Haplolobus celebicus H.J.Lam & Burseraceae & 0.92 & Low & 1 & 10 & 8 & 1.75 & 0.008 & 1 & 0.005 \\
\hline
\end{tabular}


Haplolobus floribundus (K.Schum.) H.J.Lam Haplolobus lanceolatus H.J.Lam ex Leenh.

Horsfieldia laevigata Warb.

Horsfieldia sylvestris Warb.

Horsfieldia irya (Gaertn.) Warb.

Intsia bijuga (Colebr.) Kuntze

Kokoona ochracea Merr.

Koordersiodendron pinnatum Merr.

Lepiniopsis ternatensis Valeton

Litsea ampla Merr.

Litsea firma (Blume) Hook.f.

Litsea ledermannii Teschner

Litsea timoriana Span.

Lunasia amara Blanco

Macaranga aleuritoides F.Muell

Mallotus philippensis (Lam.) Mull.Arg.

Mallotus sp.

Mastixiodendron pachyclaudos (K.Schum.) Melch.

Micromelum minutum Wight \& Arn.

Myristica fatua Houtt.

Nauclea sp.

Octomeles sumatrana Miq.

Palaquium amboinense Burck

Pimelodendron amboinicum Hassk.

Pisonia umbellifera (J.R. Forst. \& G. Forst.) Seem.

Polyalthia sumatrana (Miq.) Kurz

Polyscias nadosa (Blume) Seem.

Pometia coriacea Radlk.

Pometia pinnata J.R.Frost. \& G.Frost.

Pouteria obovata (R. Br) Baehni

Prunus costata (Hem) Kalk

Rapanea sp

Spathiostemon javensis Blume

Stemonurus javanicus Blume

Sterculia macrophylla Vent.

Sterculia parkinsonii F.Muell.

Sterculia shillinglawii F.Muell.

Sterculia sp.

Sterculia urceolata $\mathrm{Sm}$

Streblus elongatus (Miq.) Corner

Terminalia complanata K Schum.

Terminalia canaliculata Exell.

\begin{tabular}{|c|c|c|c|c|c|c|c|c|c|}
\hline Burseraceae & 0.25 & Low & $8 \pm 5.59$ & $31.49 \pm 14.51$ & $19.24 \pm 10.76$ & 5.25 & 0.089 & 2 & 0.005 \\
\hline Burseraceae & 1.22 & Low & 1.22 & $30.05 \pm 11.15$ & $17.28 \pm 11.52$ & 11.75 & 0.195 & 5 & 0.023 \\
\hline Myristicaceae & 11.09 & High & $6 \pm 2.37$ & $37.22 \pm 22.73$ & $16.90 \pm 12.08$ & 90.75 & 2.758 & 37 & 0.101 \\
\hline Myristicaceae & 0.05 & Low & 8 & 46 & 18 & 2.25 & 0.166 & 1 & 0.147 \\
\hline Myristicaceae & 3.19 & Medium & $9 \pm 4.33$ & $41.09 \pm 18.60$ & $18.51 \pm 12.57$ & 74.38 & 2.066 & 26 & 0.005 \\
\hline Leguminosae & 18.37 & High & $10 \pm 1.98$ & $58.78 \pm 13.64$ & $21.08 \pm 12.31$ & 324.83 & 15.681 & 110 & 0.381 \\
\hline Celastraceae & 0.28 & Low & $6 \pm 3.59$ & $24.07 \pm 9.93$ & $19.66 \pm 8.34$ & 4.50 & 0.049 & 2 & 0.009 \\
\hline Anacardiaceae & 1.10 & Low & $12 \pm 2.30$ & $87.07 \pm 11.59$ & $23.94 \pm 14.06$ & 24.00 & 1.705 & 6 & 0.028 \\
\hline Apocynaceae & 3.23 & Medium & $7 \pm 2.52$ & $32.01 \pm 15.19$ & $13.90 \pm 9.40$ & 45.13 & 0.980 & 20 & 0.078 \\
\hline Lauraceae & 1.14 & Low & $6 \pm 1.73$ & $30.68 \pm 11.65$ & $17.17 \pm 12.16$ & 13.25 & 0.247 & 6 & 0.028 \\
\hline Lauraceae & 0.29 & Low & 3 & 14 & 9 & 1.25 & 0.015 & 1 & 0.005 \\
\hline Lauraceae & 0.12 & Low & 5 & 21 & 13 & 1.75 & 0.035 & 1 & 0.005 \\
\hline Lauraceae & 0.06 & Low & 8 & 24 & 12 & 2.25 & 0.045 & 1 & 0.005 \\
\hline Rutaceae & 0.45 & Low & 2 & 11 & 8 & 1.25 & 0.009 & 1 & 0.005 \\
\hline Euphorbiaceae & 0.60 & Low & $3 \pm 1.79$ & $20.00 \pm 20.00$ & $9.29 \pm 7.31$ & 5.00 & 0.063 & 2 & 0.009 \\
\hline Euphorbiaceae & 0.15 & Low & 4 & 22 & 12 & 1.75 & 0.038 & 1 & 0.005 \\
\hline Euphorbiaceae & 1.82 & Low & $7 \pm 1.90$ & $24.04 \pm 15.68$ & $16.23 \pm 7.14$ & 17.00 & 0.225 & 7 & 0.032 \\
\hline Rubiaceae & 0.12 & Low & $7 \pm 4.59$ & $52.76 \pm 20.24$ & $18.21 \pm 16.79$ & 5.00 & 0.230 & 2 & 0.009 \\
\hline Rutaceae & 0.22 & Low & 4 & 16 & 12 & 2.25 & 0.020 & 1 & 0.005 \\
\hline Myristicaceae & 1.25 & Low & $6 \pm 2.71$ & $30.51 \pm 20.49$ & $18.30 \pm 12.45$ & 23.00 & 0.422 & 8 & 0.037 \\
\hline Rubiaceae & 1.40 & Low & $8 \pm 4.00$ & $71.31-9.42$ & $20.98 \pm 9.71$ & 28.75 & 2.159 & 11 & 0.046 \\
\hline Tetramelaceae & 0.08 & Low & 5 & 38 & 34 & 9.50 & 0.113 & 1 & 0.005 \\
\hline Sapotaceae & 11.00 & High & $8 \pm 2.58$ & $59.41 \pm 23.23$ & $22.92 \pm 14.12$ & 214.55 & 11.952 & 75 & 0.239 \\
\hline Euphorbiaceae & 12.28 & High & $9 \pm 2.84$ & $36.90 \pm 22.53$ & $17.34 \pm 12.66$ & 162.75 & 4.912 & 67 & 0.252 \\
\hline Nyctaginaceae & 0.07 & Low & 3 & 55 & 20 & 4.25 & 0.237 & 1 & 0.005 \\
\hline Annonaceae & 0.06 & Low & 7 & 22 & 12 & 1.75 & 0.038 & 1 & 0.005 \\
\hline Araliaceae & 0.14 & Low & 5 & 18 & 14 & 0.75 & 0.025 & 1 & 0.005 \\
\hline Sapindaceae & 23.92 & High & $9 \pm 2.44$ & $83.69 \pm 20.31$ & $24.08 \pm 13.52$ & 435.20 & 35.433 & 122 & 0.413 \\
\hline Sapindaceae & 7.34 & Medium & $10 \pm 0.98$ & $95.00 \pm 1.86$ & $22.41 \pm 9.31$ & 39.75 & 4.777 & 14 & 0.041 \\
\hline Sapotaceae & 0.75 & Low & $8 \pm 3.27$ & $45.97 \pm 21.18$ & $18.84 \pm 16.30$ & 17.50 & 0.692 & 7 & 0.032 \\
\hline Rosaceae & 5.08 & Medium & $6 \pm 2.13$ & $21.66 \pm 13.71$ & $13.63 \pm 8.93$ & 40.25 & 0.489 & 19 & 0.078 \\
\hline Primulaceae & 0.15 & Low & 5 & 13 & 12 & 1.75 & 0.013 & 1 & 0.005 \\
\hline Euphorbiaceae & 5.60 & Medium & $9 \pm 3.43$ & $39.75 \pm 27.15$ & $24.02 \pm 14.98$ & 42.58 & 0.611 & 21 & 0.087 \\
\hline Stemonuraceae & 0.27 & Low & $5 \pm 3.79$ & $26.41 \pm 23.59$ & $16.83 \pm 11.17$ & 4.00 & 0.098 & 2 & 0.009 \\
\hline Malvaceae & 4.68 & Medium & $7 \pm 2.25$ & $32.43 \pm 15.99$ & $20.46 \pm 11.43$ & 38.13 & 0.970 & 19 & 0.069 \\
\hline Malvaceae & 0.54 & Low & $7 \pm 2.74$ & $20.07 \pm 5.93$ & $23.28 \pm 7.72$ & 4.50 & 0.030 & 2 & 0.009 \\
\hline Malvaceae & 1.66 & Low & $2 \pm 1.00$ & $31.72 \pm 14.28$ & $23.76 \pm 10.90$ & 4.63 & 0.137 & 3 & 0.014 \\
\hline Malvaceae & 0.04 & Low & 9 & 38 & 14 & 1.40 & 0.113 & 1 & 0.005 \\
\hline Malvaceae & 0.16 & Low & $6 \pm 6.00$ & $31.21 \pm 29.79$ & $19.21 \pm 17.79$ & 4.75 & 0.146 & 2 & 0.005 \\
\hline Moraceae & 1.58 & Low & $8 \pm 1.28$ & $68.32 \pm 12.88$ & $21.10 \pm 14.10$ & 30.25 & 1.837 & 10 & 0.046 \\
\hline Combretaceae & 3.60 & Medium & $7 \pm 1.97$ & $45.31 \pm 13.42$ & $19.12 \pm 11.09$ & 41.63 & 1.646 & 19 & 0.014 \\
\hline Combretaceae & 0.27 & Low & $9 \pm 2.15$ & $41.42 \pm 25.25$ & $18.41 \pm 14.25$ & 6.50 & 0.272 & 3 & 0.078 \\
\hline
\end{tabular}


According to this definition, competition is an ecological process that leads to interactions between individuals and has an effect on reducing the survival, growth and reproduction potential of competing individuals (Begon et al. 1986; Maleki et al. 2015). Competition is caused by the limited supply of resources which can support optimal growth of two or three trees (Brand and Magnussen 1988; Holmes and Reed 1991; Gadow and Hui 1999; Pretzsch 2002; Rivas et al. 2005), leading to interference reaction between individuals (Begon et al. 1986; Bazzaz 1990; Goldberg 1990; Teughels et al. 1995). Resources that trigger competition are nutrients and water, and light and space to flourish (Teughels et al. 1995; Tschirhart 2001).

There is no significant relationship between the position of the competitor and Hegyi index. The Hegyi index and the total height, the canopy area shows a significant negative correlation $(p<0.01)$ (Table 5).

The position of competitor plants is an important factor in evaluating the characteristics of competition among merbau trees in GMNTPM as it closely related to the need of growth space on the soil surface. Within its relation to the sunlight direction, the photosensitivity might also reduce the effect of the plant position toward the competitive intensity. Therefore, in this research, the position of competitor plants was regarded as a complementary factor of other factors which included the distance and dendrometric factors which also influence the level of competition among merbau trees as well as competition between merbau trees and other plants. This assumption is also closely related to the topography of GMNTPM. Thus, generally, regardless of where competitor plants grow, the amount of sunlight obtained by merbau leaves would not be reduced. However, rocky soil creates gaps in the canopy, resulting in lower canopy coverage.

The number of stands, number of species, tree height, diameters, and canopy cover of competitors were relatively the same in all directions and in general, they were directly proportional to the intensity of the competition (Table 4). The position of the competitors did not have a direct functional relationship with the diversity and structure of the competitor's structure. In this context, the main focus is the forest structures. Hence, the effect of tree layout toward competition intensity indicated whether GMNTPM is a primary forest which has reached the homeostatic condition. Fraver et al. (2014) reported that continuous competition affected the process and structure of a forest. They also found that plant positioning strongly influenced the growth rate of Picea abies as it triggered tighter competition. However, according to Berube-Deschenes et al. (2017), within the context of distance-dependent competition, tree positioning is a useful aspect that characterizes the acquisition of potential resources and integration of stand structural variation. However, according to Berube-Deschenes et al. (2017) in distancedependent competition, the position of the tree is useful for characterizing the acquisition of potential resources and integrating the structural variation of stands.
Competitor position was a function of structural adaptation to the craggy soil condition and density of stands in GMNTPM. The growing position of neighboring trees was the beginning of competition, because it was closely related to where and what species of competitors growing alongside the merbau population. The correlation shows the spatial arrangement by morphological adaptation because if the competition intensity is high, the height growth and canopy width of the competing trees tend to be inhibited (Table 5). It is related to the fact that the position of the competitors (Table 4) limits the growth space and potential sunlight received by merbau (intraspecific competition). The position of the competitors is not taken into account in the Hegyi index, but with the quadrant approach that we used in data collection, the position of the competitors become quite important due to ecological reasons.

Table 2. Mean of distance, canopy cover, and Hegyi index of the most competitive trees species (MCTS)

\begin{tabular}{lccc}
\hline DBH class & $\begin{array}{c}\text { Mean of } \\
\text { distance }(\mathbf{m})\end{array}$ & $\left.\begin{array}{c}\text { Mean of } \\
\text { canopy cover }\end{array} \mathbf{( m}^{\mathbf{2}}\right)$ & $\begin{array}{c}\text { Mean of } \\
\text { Hegyi index }\end{array}$ \\
\hline$<10 \mathrm{~cm}$ & 5.69 & 2.59 & 0.60 \\
$10-19 \mathrm{~cm}$ & 3.65 & 1.73 & 0.43 \\
$20-29 \mathrm{~cm}$ & 5.10 & 2.26 & 0.20 \\
$30-39 \mathrm{~cm}$ & 5.30 & 2.78 & 0.13 \\
$40-49 \mathrm{~cm}$ & 6.33 & 3.40 & 0.08 \\
$\geq 50 \mathrm{~cm}$ & 6.60 & 4.75 & 0.07 \\
\hline
\end{tabular}

Table 3. Correlation between Hegyi index with total height and canopy cover of the most competitive trees species (MCTS)

\begin{tabular}{llccc}
\hline & & $\begin{array}{c}\text { Hegyi } \\
\text { index }\end{array}$ & $\begin{array}{c}\text { Total } \\
\text { height }\end{array}$ & $\begin{array}{c}\text { Canopy } \\
\text { cover }\end{array}$ \\
\hline Hegyi & Pearson correlation & 1 & $-.193^{* *}$ & $-.207^{* *}$ \\
index & Significance (2-tailed) & & .000 & .000 \\
& $\mathrm{~N}$ & 655 & 654 & 655 \\
Total & Pearson correlation & $-.193^{* *}$ & 1 & $.596^{* *}$ \\
height & Significance (2-tailed) & .000 & & .000 \\
& $\mathrm{~N}$ & 654 & 654 & 654 \\
Canopy & Pearson correlation & $-.207^{* *}$ & $.596^{* *}$ & 1 \\
cover & Significance (2-tailed) & .000 & .000 & \\
& $\mathrm{~N}$ & 655 & 654 & 655 \\
\hline
\end{tabular}

Note: **. Correlation is significant at the 0.01 level (2-tailed)

Table 4. Hegyi index, distance and stand structure, competitor dendrometric factor according to growing position

\begin{tabular}{|c|c|c|c|c|c|c|c|}
\hline \multirow[t]{2}{*}{ Position } & $\begin{array}{c}\text { Mean } \\
\text { of }\end{array}$ & $\underset{s}{\Sigma}$ & $\underset{\text { snecies }}{\Sigma}$ & $\begin{array}{c}\text { Mean } \\
\text { of }\end{array}$ & \multirow{2}{*}{$\begin{array}{c}\text { Mean } \\
\text { of } \\
\text { DBH } \\
\text { (cm) }\end{array}$} & \multirow{2}{*}{$\begin{array}{c}\text { Mean } \\
\text { of } \\
\text { total } \\
\text { height } \\
\text { (m) }\end{array}$} & \multirow{2}{*}{$\begin{array}{c}\text { Mean } \\
\text { of } \\
\text { canopy } \\
\text { cover } \\
\left(\mathbf{m}^{2}\right)\end{array}$} \\
\hline & $\begin{array}{l}\text { Hegyi } \\
\text { index }\end{array}$ & & & $\begin{array}{c}\text { distance } \\
\text { (m) }\end{array}$ & & & \\
\hline East & 0.17 & 106 & 30 & 5.42 & 34.79 & 15.96 & 2.73 \\
\hline North & 0.20 & 110 & 42 & 5.04 & 34.93 & 15.95 & 2.70 \\
\hline Northeast & 0.19 & 110 & 33 & 5.58 & 37.25 & 16.65 & 2.81 \\
\hline Northwest & 0.15 & 110 & 36 & 5.31 & 38.49 & 16.85 & 3.01 \\
\hline South & 0.25 & 96 & 36 & 5.54 & 32.83 & 16.60 & 2.69 \\
\hline Southeast & 0.16 & 120 & 36 & 5.23 & 34.22 & 16.38 & 2.97 \\
\hline Southwest & 0.19 & 94 & 32 & 5.16 & 32.97 & 16.44 & 2.72 \\
\hline West & 0.15 & 103 & 33 & 5.40 & 34.65 & 15.45 & 2.75 \\
\hline
\end{tabular}


Table 5. Correlation of Hegyi index with tree position, total height, canopy cover of competitors

\begin{tabular}{|c|c|c|c|c|c|}
\hline & & $\begin{array}{l}\text { Hegyi } \\
\text { index }\end{array}$ & Position & $\begin{array}{c}\text { Total } \\
\text { height }\end{array}$ & $\begin{array}{c}\text { Canopy } \\
\text { cover }\end{array}$ \\
\hline \multirow[t]{3}{*}{$\begin{array}{l}\text { Hegyi } \\
\text { index }\end{array}$} & $\begin{array}{l}\text { Pearson } \\
\text { correlation }\end{array}$ & 1 & -.025 & $-.202^{* *}$ & $-.218^{* *}$ \\
\hline & $\begin{array}{l}\text { Significance (2- } \\
\text { tailed) }\end{array}$ & & .471 & .000 & .000 \\
\hline & $\mathrm{N}$ & 849 & 849 & 848 & 849 \\
\hline \multirow[t]{3}{*}{ Position } & $\begin{array}{l}\text { Pearson } \\
\text { correlation }\end{array}$ & -.025 & 1 & -.012 & .011 \\
\hline & $\begin{array}{l}\text { Significance (2- } \\
\text { tailed) }\end{array}$ & .471 & & .721 & .739 \\
\hline & $\mathrm{N}$ & 849 & 849 & 848 & 849 \\
\hline \multirow[t]{3}{*}{$\begin{array}{l}\text { Total } \\
\text { height }\end{array}$} & $\begin{array}{l}\text { Pearson } \\
\text { correlation }\end{array}$ & $-.202^{* *}$ & -.012 & 1 & $.570^{* *}$ \\
\hline & $\begin{array}{l}\text { Significance (2- } \\
\text { tailed) }\end{array}$ & .000 & .721 & & .000 \\
\hline & $\mathrm{N}$ & 848 & 848 & 848 & 848 \\
\hline \multirow[t]{3}{*}{$\begin{array}{l}\text { Canopy } \\
\text { cover }\end{array}$} & $\begin{array}{l}\text { Pearson } \\
\text { correlation }\end{array}$ & $-.218^{* *}$ & .011 & $.570^{* *}$ & 1 \\
\hline & $\begin{array}{l}\text { Significance (2- } \\
\text { tailed) }\end{array}$ & .000 & .739 & .000 & \\
\hline & $\mathrm{N}$ & 849 & 849 & 848 & 849 \\
\hline
\end{tabular}

Mueller-Ellenberg and Dombois (1974), Weiner at al. (1990) explains that competition of two species of plants with the same form of growth starts from the same way of adaptation to environmental factors. In other words, competition is mediated by growth factors. The spatial pattern is the visualization of adaptation to certain environmental factors. Therefore, the spatial pattern is closely related to the multi-species coexistence aspect (Nakagawa et al. 2015; Callaway 1995; Condit et al. 2000; Stoll and Prati 2001; Murrell 2009) such as the competition between trees that depends on spatial relationships between plants and neighbors to get resources (Teughels et al. 1995).

The position of the competitors has a direct effect on the dendrometric aspects of competitors and merbau trees which ultimately has an impact on competition. When the position of each competitor in each sampling unit does not change due to the death of the stand, then over time, the intensity of the competition will increase because space becomes narrower due to the growing dimension of the stands of each individual, both competitors and merbau trees. According to Tschirhart (2001), Tremmel and Bazzaz (1993), each plant community occupies a fixed space and when each stand increases its biomass due to growth or because it produces new plants, space will be filled. When space is filled, each stand that increases its biomass will have difficulty in absorbing energy because of the shadow of other stands. This condition is a negative adverse effect because the canopy of neighboring trees blocks potential energy.

\section{The effect of competition on merbau}

The intensity of competition produces two forms of process behavior, namely the negative effects of individual neighbors and the response of trees to changes in resource abundance (Teughels et al. 1995; Goldberg 1990; Tilman 1990; Bazzaz and McConnaughay 1992). The effect of competition can be defined mechanically as the influence of plants around the resource. The competition response can be seen as a relationship between the number of resources available to plants and some fitness components such as growth, survival and reproductive output (Goldberg 1990).

In this study, merbau experienced the effects of intraspesific and interspecific competition. Intraspecific effects of competition occurred because it grew in groups or cluster pattern. Cluster patterns of species are affected by dispersal limitations at larger scales (Burslem et al. 2001; Ledo et al. 2014). The restriction of merbau distribution was due to the relatively large and heavy seed size, so it did not support broad spreading farther from the stand of the mother tree, either with the help of wind or water. At GMNTPM, merbau also did not have any frugivores that could help to spread the seeds that physiologically had been mature. These factors caused merbau to grow in clusters under the stand of its mother tree.

Merbau seeds could fell far from the mother tree due to mechanical factors of the mother tree and neighboring trees. Seeds that escape from the pods bounce off the branches or the branches of neighboring trees so that it fell far from the stand of the mother tree. If this mechanism occurred to the ripe fruit that hung at the end of the longest branch, the initial stage of merbau invading more extensive areas at GMNTPM. However, generally, the first process occurred in a much smaller frequency than the process of the merbau seeds falling and germinating and forming regeneration groups under and around the mother tree.

The pattern of merbau grouping occurred since seed germination phase, so regeneration density caused the distance between individuals to be quite close. As a result, space for growth was limited to the effect of same-species competition. According to Del Rio et al. (2014), in general, the more limited the growing space by neighbors, the stronger the competition for resources for individual growth. The results of research by Fraver et al. (2014) showed that the position of Picea abies affected the intensity of competition growth with the canopy as a measure of assessment. The intensity of competition is increasing and influences the level of growth if the tree position tends to be a clustering pattern, while the effect of competition is smaller if the pattern is not a cluster.

The competition due to tree density causes growing space for one of the competing parties to decrease and there is the light interception by neighboring trees (Tremmel and Bazzaz 1993, 1995; Mori and Takeda 2003). The effects of this pattern of competition are negative density-dependent (NDD) because density reduces the number of stands due to competition (Wright 2002; Piao et al. 2014).

At the population level, NDD produces a self-thinning mechanism because resources are limited so that some individuals are die in response to competition (Morris 2002, 2003; Chu et al. 2010; Harper 1977; Bazzaz 1996; Lentz 1999). The reason for all same-species stands, every 
individual competes with similar behavior. Therefore, space and resources are insufficient for all. It is related to tree size and homogeneous genetic factors (Pretzsch 2014). Thus, several studies explain that the effect of competition through conspecific behavior is more significant on individual growth compared to the effects of heterospecific neighbors (Comita et al. 2010; Johnson et al. 2012; Zhu et al. 2015). Zhu et al. (2015) explains that in tropical forests, the NDD conspecific effects on local tree species are more significant in the early stages of growth and the effects are smaller in the adult stage. Larson et al. (2015) found that in Abies amabilis young forests, the mortality rate per year due to density-dependent competition is higher than the old forest, causing more extensive distances between trees. This condition is called competition turns into facilitation so that the surviving trees can develop insufficient space and new variations are formed in the stand structure. Clarified by Kunstler et al. (2016) that the fast maximum growth of a species is negatively correlated with stand density in all biomes, and correlated positively with specific leaf area in most biomes.

Direct indication of NDD mechanism that produces self-thinning was not found as the samples of merbau poles involved in this research was the young ones which had been able to survive in GMNTPM. However, the results of research was done by Sirami (2014), Sadono et al. (2014) in the same location confirmed the existence of the direct indication of NDD mechanism that produces self-thinning. They found out that merbau trees produced a massive amount of seedlings under the parent trees. However, neither saplings or poles were often not found. This phenomenon normally occurs in merbau stands structure in the low land forest of Papua (Forestry Service of Papua Province 2008, 2010a, 2010b).

NDD with self-thinning mechanism because of the competition were found in the development of the merbau population at GMNTPM. Signs that merbau will survive into an adult tree, generally can be seen when it has reached the sapling phase with a diameter of $>2 \mathrm{~cm}$ (Sirami 2014; Sadono et al. 2014). In this diameter, merbau has shown relatively wide spacing of germinations compared to seedling phases which are directly clustered under the mother trees. These facts confirm that the interspecific competition in the merbau population at GMNTPM is a strong indication of the need for ideal spacing for merbau on cultivated land, an important factor that can support its growth.

The interspecific competition was occurred between merbau and 79 other tree species. They could adapt well on rough soil and occupied strata A-C at GMNTPM (Table 1). Strata $C$ was the densest canopy layer because it was not only inhabited by tree species but also several species of lianas (Sirami et al. 2016) and herbs from the family of Arecaceae and Pandanaceae. Generally, trees in strata C were smaller in diameter, so that they could grow at a closer distance. While the trees in the strata A and B had a relatively larger diameter, so the distance was wider. It was recorded that more than 500 competitor stands occupied the $\mathrm{C}$ strata, while the rests were large trees in strata A and B.

The factors that cause the variation in the intensity of competition between merbau and competitors are the average distance and diameter as the theoretical basis of the Hegyi Index. However, there are also other factors such as frequency of presence, total height, canopy cover, and the number of stands that indirectly affect the intensity of competitors but they are not covered in Hegyi's index.

A significant positive relationships are shown between Hegyi index with the frequency of presence, number of stands, total height and canopy area $(p<0.01)$ (Table 6). If there is an increase in the number of individuals, the total height, canopy area and frequency of a competitor, the intensity of competition with merbau will increase.

Table 6. Correlation between Hegyi index, frequency, number of stands, total height, canopy cover of competitors

\begin{tabular}{|c|c|c|c|c|c|c|}
\hline & & Hegyi index & Frequency & Number of stands & Total height & Canopy cover \\
\hline \multirow[t]{3}{*}{ Hegyi index } & Pearson correlation & 1 & $.942^{* *}$ & $.970^{* *}$ & $.931^{* *}$ & $.932^{* *}$ \\
\hline & Significance (2-tailed) & & .000 & .000 & .000 & .000 \\
\hline & $\mathrm{N}$ & 80 & 80 & 80 & 80 & 80 \\
\hline \multirow[t]{3}{*}{ Frequency } & Pearson correlation & $.942^{* *}$ & 1 & $.970^{* *}$ & $.979^{* *}$ & $.972^{* *}$ \\
\hline & Significance (2-tailed) & .000 & & .000 & .000 & .000 \\
\hline & $\mathrm{N}$ & 80 & 80 & 80 & 80 & 80 \\
\hline \multirow[t]{3}{*}{ Number of stands } & Pearson correlation & $.970^{* *}$ & $.970^{* *}$ & 1 & $.966^{* *}$ & $.963^{* *}$ \\
\hline & Significance (2-tailed) & .000 & .000 & & .000 & .000 \\
\hline & $\mathrm{N}$ & 80 & 80 & 80 & 80 & 80 \\
\hline \multirow[t]{3}{*}{ Total height } & Pearson correlation & $.931^{* *}$ & $.979^{* *}$ & $.966^{* *}$ & 1 & $.995^{* *}$ \\
\hline & Significance (2-tailed) & .000 & .000 & .000 & & .000 \\
\hline & $\mathrm{N}$ & 80 & 80 & 80 & 80 & 80 \\
\hline \multirow[t]{3}{*}{ Canopy cover } & Pearson correlation & $.932^{* *}$ & $.972^{* *}$ & $.963^{* *}$ & $.995^{* *}$ & 1 \\
\hline & Significance (2-tailed) & .000 & .000 & .000 & .000 & \\
\hline & $\mathrm{N}$ & 80 & 80 & 80 & 80 & 80 \\
\hline
\end{tabular}

Note: **. Correlation is significant at the 0.01 level (2-tailed). 
The size of the diameter and height of the competitors indicate the growing space of above-ground that is controlled by a tree. The canopy area when connected with the height of the tree is a description of the increasing space in the forest canopy which is controlled by a tree.

Competitors monopolized two levels of above-ground space. It reflected the intensity of the merbau competition with neighboring trees. Temporarily, the dendrometric elements were a function of tree architecture that was always dynamic. Increasing the height and size of the crown is the effort of the competitors to reach spaces with dimensions above the ground surface that provides optimum sunlight. Ford (2014) explains that competition is closely related to the dynamics of tree architecture because when there is a change in plant architecture, it changes the surrounding environment. Therefore, it turns the available resources for the plant and its neighbors. The dendrometric factor is closely related to the adaptation of tree parts to support the ability to compete with neighboring trees. The expansion of tree architecture aims to position their leaves between their neighbors and sources of light by growing taller, earlier, faster, or all three (Craine and Dybzinski 2013; Aschehoug et al. 2016). Therefore, key characteristics of the light competition include phenology, height, and relative growth rates (Aschehoug et al. 2016). These characteristics indicate that light competition is an asymmetrical competition because larger plants get disproportionately larger portions of light.

The growth dynamics of every element of tree architecture such as height, $\mathrm{DBH}$, and canopy cover have long been considered as dendrometric factors that determine the intensity of competition between trees. Several previous studies have proven that $\mathrm{DBH}$ is an important factor that influences the intensity of competition between trees both interspecific and intraspecific competition (Gonzalez de Andres et al. 2018; Zhou 2017; Da Cunha et al. 2016).

The competitors that grow closer to merbau increase the intensity of competition because the total growth space needed is higher. The effect of the number of trees on the intensity of the competition will be even greater, if it is followed by a fairly close distance and large diameter and growing position around merbau. Previous researchers have explained the density effect that the relationship of plant density changes available resources, thus creating competition (Bonan 1991; Chu et al. 2009; Chu et al. 2010).

In tropical forests with high tree species diversity such as GMNTPM, there are two mechanisms of competition at once namely conspecific and heterospecific. During field observations, most of the competitors grew in groups per species, but only the closest stands located near merbau were recorded for analysis. Furthermore, there was a mixture of heterospecific distribution patterns. Not just being a competitor for merbau, some of the species of competitors also experience competition in their population and with other species other than merbau. This condition is an indication that the density of stands and spatial patterns are important factors that determine the high intensity of competition with merbau trees.

Competition with merbau can be considered as the effect of competition between competitors with nonmerbau species. For competitors which have a faster growth rate than merbau may choose to be more dominant in achieving light resources on the side of merbau than on the side of same-species trees or with other species that have relatively same growth speed. This condition is an indicator of the effects of heterospecific and conspecific mechanisms that occur together.

The frequency of the presence of a species around merbau can increase the intensity of the competition in species level. However, it also should meet other conditions such as large diameter and more than one individual. The frequency associated with the heredity factor explains that each species carries different genetic identities that affect how the species grows and develops in the dense forest communities. Bennett et al. (2016) demonstrate that each species has a different effect on its competition with other species. This condition is one of the ecological processes that show how heredity works. In this study, competitor species with the highest competition intensity also had a high frequency of presence (Table 1) and were relatively dominant. Domination is another indication of the ability to compete because the species can grow on stand structures that are higher than other species. Those competitor species belong to the group of most competitive tree species.

\section{Merbau's response to competition}

The canopy architecture and canopy slope of merbau is different from the competitor species. Merbau tends to adapt irregular canopy shapes than oval, round, triangular and domed (Table 7). The irregular canopy shapes were formed when merbau's canopy had direct contact with the competitor's canopy. Round, oval, triangular or domes shapes occurred when the merbau's canopy was in the gap between competitors' canopies. Merbau also tended to tilt the canopy in a different direction from that of competitors and grew the first branch at the lowest point on the trunk compared to the competitors.

This behavior is contrary to the growth behavior of Pometia spp., Dysoxylum spp., and Dracontomelon sp. in the pole phase, these three species do not grow branches at the lowest point on the stem until they reach a relatively open space between the other tree canopies. This strategy is aimed to minimize the horizontal barrier because at the lowest position merbau tree branches can grow in less dense spaces. On the rough soil conditions in GMNTPM, soil cover and herbaceous plants are rarely to be found, so there is enough space near the soil surface. This condition allows merbau leaves on the lowest branches to be free from the barrier to absorb sunlight because the size of the merbau leaves is smaller than that of the competitors in general. 
Table 7. Canopy shape, canopy slope, and height of merbau tree branches

\begin{tabular}{lc}
\hline Item & Percentage \\
\hline Canopy shape & \\
Irregular & $82 \%$ \\
Oval & $11 \%$ \\
Round & $5 \%$ \\
Triangular & $2 \%$ \\
& \\
Canopy slope direction & \\
Very different & $60.55 \%$ \\
Different & $31.19 \%$ \\
Quite different & $5.50 \%$ \\
Less different & $0.92 \%$ \\
Same & $0.46 \%$ \\
& \\
Lowest branch height & \\
The tallest & $6.05 \%$ \\
High & $4.63 \%$ \\
Quite high & $1.78 \%$ \\
Low & $16.37 \%$ \\
The lowest & $48.04 \%$ \\
\hline
\end{tabular}

Merbau responded to the high intensity of competition through several growing behaviors (Table 7). This form of response is part of the ability to compete as well as a merbau strategy to absorb light among the competing tree canopies at GMNTPM. Light is a limited resource under the tree canopy in tropical forests (Chazdon and Fetcher 1984; Chazdon and Pearcy 1991; Guzman and Cordero 2016), thus, light availability affects some plant characters and contributes to the coexistence of plants in different habitats (Hubbell et al. 1999; Adler et al. 2013). A character affected by light is the canopy architecture, such as shape and width. Therefore, when the light is limited, competition between large trees and small trees under the canopy often shows partial size asymmetry (Schwinning and Weiner 1998; Looney et al. 2016).

What merbau did as a response to competition, has been investigated by ecologists. They argue that the adaptive needs of tree architecture are related to the efficiency of capturing light, such as the position of branches which are right above the ground affect the pattern of leaf appearance (Wickens and Horn 1972; Sakai 1986; Kohyama 1987; Takenaka 1994). Therefore, the success in competition is also determined by the placement of leaves on the canopy because it is very important to capture light (Black 1958, 1960; Tremmel and Bazzaz 1995). It compensates the effects of competition through architectural and physiological plasticity (Grace 1995) by positioning the leaves among its neighbors and light sources (Craine and Dybzinski 2013; Aschehoug et al. 2016).

However, light competition can be quite complicated to understand because shade avoidance syndrome (SAS) can occur in areas with abundant light (Pierik et al. 2013; Aschehoug et al. 2016). In this research, three samplings of merbau aborted the leaves even though they grew under the competitors' canopy. Whether this is another form of SAS or not, it needs to be investigated further because merbau is a semi-tolerant species.

\section{Ideal planting space}

The width of the canopy of adult merbau trees can reach $>600 \mathrm{~m}^{2}$ (Sadono et al. 2014; Sirami 2014). Merbau also tends to adapt its canopy slopes with different directions, has irregular canopy shapes and the lowest clear bole height among competitors. In silviculture, the growth behavior is closely related to the effect of the canopy on the diameter size and the total height of trees that can be harvested. Thus, by setting the ideal planting space and considering the width of the canopy of each species in nature, it will reduce the intensity of competition in the merbau plantations.

Based on merbau growing behavior and $\mathrm{DBH}$ grouping of the most competitive trees species, we concluded that ideal planting space between merbau and shade trees is 3-7 $\mathrm{m}$. If the purpose of planting is for the use of wood with a DBH of 10-30 cm, the planting space ranges from 3 to $5 \mathrm{~m}$. While, if the object is for the use of wood with a DBH of $>$ $30 \mathrm{~cm}$ or for $30-40$ years of rotation, the planting space is between $5-7 \mathrm{~m}$.

\section{ACKNOWLEDGEMENTS}

We gratefully acknowledge all the members of the field team who have worked hard during data collection at the Gunung Meja Nature Tourism Park of Manokwari. Special thanks go to the honorable anonymous reviewers who have been willing to revise our manuscripts.

\section{REFERENCES}

Aarssen LW, Schamp BS, Wight S. 2014. Big plants do they affect neighbourhood species richness and composition in herbaceous vegetation? Acta Oecol 55: 36-42.

Adler PB, Fajardo A, Kleinhesselink AR, Kraft NJB. 2013. Trait-based tests of coexistence mechanisms. Ecol Lett 16: 1294-1306.

Amiri M, Naghdi R. 2016. Assessment of competition indices of an unlogged oriental beech mixed stand in Hyrcanian forests, Northern Iran. Biodiversitas 17 (1): 306-314.

Aschehoug ET, Brooker R, Atwater DZ, Maron JL, Callaway RM. 2016. The mechanisms and consequences of interspecific competition among plants. Ann Rev Ecol Evol Syst 47: 263-281.

Barua SK, Lehtonen P, Pahkasalo T. 2014. Plantation vision: potentials, challenges and policy options for global industrial forest plantation development. Intl For Rev 16: 117-127.

Bazzaz FA, McConnaughay KDM. 1992. Plant plant interactions in elevated $\mathrm{CO}_{2}$ environments. Australian J Bot 40 (5): 547-563.

Bazzaz FA. 1990. Plant-plant interactions in successional environments. In: Grace JB and Tilman D (Eds) Perspectives on Plant Competition. Academic Press Inc, London.

Bazzaz FA. 1996. Plants in Changing Environments: Linking Physiological, Population, and Community Ecology. Cambridge University Press, Cambridge.

Begon M, Harper JL, Townsend CR. 1986. Ecology, Individuals, Populations and Communities. Blackwell Scientific Publications, Oxford.

Bennett JA, Riibak K, Tamme R, Lewis RJ, Partel M. 2016. The reciprocal relationship between competition and intraspecific trait variation. J Ecol 104:1410-1420.

Berube-Deschenes A, Franceschini T, Schneider R. 2017. Quantifying competition in white spruce (Picea glauca) plantations. Ann For Sci 74: 26.

Black JN. 1958. Competition between plants of different initial seed sizes in swards of subterranean clover (Trifolium subterraneum L.) with 
particular reference to leaf area and the light microclimate. Australian J Agric Res 9 (3): 299-318.

Black JN. 1960. The significance of petiole length, leaf area, and light interception in competition between strains of subterrranean clove (Trifolium subterraneum L.) grown in swards. Australian J Agric Res 11 (3): 277-291.

Bonan GB. 1991. Density effects on the size structure of annual plan populations: an indication of neighborhood competition. Ann Bot 68 (4): 341-347.

Brand DG, Magnussen S.1988. A symmetric, two-sided competition in even-aged monocultures of red pine. Canadian J For Res 18: 901-910.

Bremer LL, Farley KA. 2010. Does plantation forestry restore biodiversity or create green deserts? a synthesis of the effects of land-use transitions on plant species richness. Biodiv Cons 19: 3893-3915.

Burkhart HE, Tome M. 2012. Modeling Forest Trees and Stands. Springer, Dordrecht.

Burslem DFRP, Garwood NC, Thomas SC. 2001. Tropical forest diversity the plot thickens. Sci 292: 606-607.

Callaway RM. 1995. Positive interactions among plants. Bot Rev 61: 306 349.

Chazdon RL, Fetcher N. 1984. Photosynthetic light environments in a lowland tropical rain forest in Costa Rica. J Ecol 72: 553-564.

Chazdon RL, Pearcy RW. 1991. The importance of sunflecks for forest understory plants. Biosci 41: 760-766.

Chu CJ, Weiner J, Maestre FT, Wang YS, Morris C, Xiao S, Yuan JL, Du GZ, Wang G. 2010. Effects of positive interactions, size symmetry of competition and abiotic stress on self-thinning in simulated plant populations. Ann Bot 106 (4): 647-652.

Chu CJ, Weiner J, Maestre FT, Xiao S, Wang YS, Li Q, Yuan JL, Zhao LQ, Ren ZW, Wang G. 2009. Positive interactions can increase size inequality in plant populations. J Ecol 97: 1401-1407.

Clark III A, Jordan L, Schimleck L, Daniels RF. 2008. Effect of initia planting spacing on wood properties of unthinned loblolly pine at age 21. For Prod J 58 (10): 78-83.

Collins B, Wein G. 2000. Stem elongation response to neighbor shade in sprawling and upright Polygomun species. Ann Bot 86 (4): 739-744.

Comita LS, Muller-Landau HC, Aguilar S, Hubbell SP. 2010 Asymmetric density dependence shapes species abundances in a tropical tree community. Sci 329: 330-332.

Condit R, Ashton PS, Baker P, Bunyavejchewin S, Gunatilleke S, Gunatilleke N, Hubbell SP, Foster RB, Itoh A, LaFrankie JV, Lee HS, Losos E, Manokaran N, Sukumar R, Yamakura T. 2000. Spatial patterns in the distribution of tropical tree species. Sci 288: 14141418.

Contreras MA, Affleck D, Chung W. 2011. Evaluating tree competition indices as predictors of basal area increment in Western Montana Forests. For Ecol Manag 262: 1939-1949.

Coomes DA, Allen RB. 2007. Effects of size, competition, and altitude on tree growth. J Ecol 95: 1084-1097.

Craine JM, Dybzinski R, 2013. Mechanisms of plant competition: mechanisms of plant competition for nutrients, water and light. Func Ecol 27: 833-840.

Da Cunha TA, Finger CAG, Hasenauer H. 2016. Tree basal area increment models for Cedrela, Amburana, Copaifera, and Swietenia growing in the Amazon rain forests. For Ecol Manag 365: 174-183.

Del Rio M, Schutze G, Pretzsch H. 2014. Temporal variation of competition and facilitation in mixed species forests in Central Europe. Plant Bio 16: 166-176.

Ford ED. 2014. The dynamic relationship between plant architecture and competition. Front Plant Sci 5 (275): 1-13.

Forestry Service of Papua Province. 2008. Inventory and Survey of Merbau (Intsia spp.) Species at HPH PT. Hanurata, Senggi District, Keerom Regency, Papua Province. Research Report of Forestry Service of Papua Province, Jayapura. [Indonesian]

Forestry Service of Papua Province. 2010a. Inventory of Standing Stock of Merbau (Intsia spp.) in Mappi Regency, Papua Province. Research Report of Forestry Service of Papua Province, Jayapura. [Indonesian]

Forestry Service of Papua Province. 2010b. Inventory of Standing Stock of Merbau (Intsia spp.) in Mamberamo Raya Regency, Papua Province. Research Report of Forestry Service of Papua Province, Jayapura. [Indonesian]

Fraver S, D'Amato AW, Bradford JB, Jonsson BG, Jonsson M, Esseen PA 2014. Tree growth and competition in an old-growth Picea abies forest of boreal Sweden: influence of tree spatial patterning. J Veg Sci 25: $374-385$
Gadow Kv, Hui GY. 1999. Modelling Forest Development. Kluwer Academic Publishers, Dordrecht.

Goldberg DE. 1990. Components of resource competition in plant communities. In: Grace JB and Tilman D (Eds) Perspectives on Plant Competition. Academic Press Inc., London.

Goldberg DE. 1996. Competitive ability: definition, contingency, and correlated traits. Phil Trans R Soc B 351: 1377-1385.

Gonzalez de Andres E, Camarero JL, Blanco JA, Imbert JB, Lo YH, Sanguesa-Barreda G, Castillo FJ. 2018. Tree-to-tree competition in mixed European beech-Scots pine forests has different impacts on growth and water-use efficiency depending on site conditions. J Ecol 106: $59-75$

Grace JB. 1990. On the relationship between plant straits and competitive ability. In: Grace JB, Tilman D, (eds) Perspectives on Plant Competition. Academic Press, London.

Grace JB.1995. On the measurement of plant competition intensity. Ecol 76: 305-308.

Grime JP. 1979. Plant Strategies and Vegetation Processes. John Wiley \& Sons, New York.

Guzman QAJ, Cordero SRA. 2016. Neighborhood structure influences the convergence in light capture efficiency and carbon gain: an architectural approach for cloud forest shrubs. Tree Physiol 36: 712724.

Harper JL. 1977. Population Biology of Plants. Academic Press, New York.

Holmes MJ, Reed DD. 1991. Competition indices for mixed species Northern Hardwoods. For Sci 37 (5): 1.338-1.349.

Hubbell SP, Foster RB, O’Brien ST, Harms KE, Condit R, Wechsler B, Wright SJ, Loo de Lao S. 1999. Light-gap disturbances, recruitment limitation, and tree diversity in a neotropical forest. Sci 283: 554-557.

Jacovelli PA. 2014. The future of plantations in Africa. Intl For Rev 16: 144-159.

Johnson D, Beaulieu WT, Bever JD, Clay K. 2012. Conspecific negative density dependence and forest diversity. Sci 336: 904-907.

Kohyama T. 1987. Significance of architecture and allometry in saplings. Func Ecol 1: 399-404.

Kunstler G, Albert CH, Courbaud B, Lavergne S, Thuiller W, Vieilledent G, Zimmermann NE, Coomes DA. 2011. Effects of competition on tree radial growth vary in importance but not in intensity along climatic gradients. J Ecol 99: 300-312

Kunstler G, Falster D, Coomes DA, Hui F, Kooyman RM, Laughlin DC, Poorter L, Vanderwel M, Vieilledent G, Wright SJ, Aiba M, Baraloto C, Caspersen J, Cornelissen JHC, Gourlet-Fleury S, Hanewinkel M, Herault B, Kattge J, Kurokawa H, Onoda Y, Penuelas J, Poorter H, Uriarte M, Richardson S, Ruiz-Benito P, Sun IF, Stahl G, Swenson NG, Thompson J, Westerlund B, Wirth C, Zavala MA, Zeng H, Zimmerman JK, Zimmermann NE, Westoby M. 2016. Plant functional traits have globally consistent effects on competition. Nat 529: 204-207.

Larson AJ, Lutz JA, Donato DC, Freund JA, Swanson ME, Hillerislambers J, Sprugel DG, Franklin JF. 2015. Spatial aspects of tree mortality strongly differ between young and old-growth forests. Ecol 96 (11) : 2855-2861

Ledo A, Canellas I, Barbeito I, Gordo FJ, Calama RA, Gea-Izquierdo G. 2014. Species coexistence in a mixed Mediterranean pine forest: Spatio-temporal variability in trade-offs between facilitation and competition. For Ecol Man 322: 89-97.

Lentz KA. 1999. Effects of intraspecific competition and nutrient supply on the endangered Northeastern Bulrush, Scirpus anchistrocaetus Schuyler (Cyperaceae). Amer Mid Nat 142 (1): 47-54.

Looney CE, D’Amato AW, Fraver S, Palik BJ, Reinikainen MR. 2016. Examining the influences of tree-to-tree competition and climate on size-growth relationships in hydric, multi-aged Fraxinus nigra stands. For Ecol Man 375: 238-248.

MacArthur R, Levins R. 1967. The limiting similarity, convergence, and divergence of coexisting species. Amer Nat 101: 377-385.

Maleki K, Kiviste A, Korjus H. 2015. Analysis of individual tree competition effect on diameter growth of silver birch in Estonia. For Sys 24 (2): 1-13.

Margono BA, Potapov PV, Turubanova S, Stolle F, Hansen MC. 2014. Primary forest cover loss in Indonesia over 2000-2012. Nat Clim Ch 4: $730-735$

Marler TE. 2015. Balancing growth and wood quality of Intsia bijuga under management: complexicity of silviculture conservation decision. J Trop For Sci 27: 427-432. 
Mori A, Takeda H. 2003. Architecture and neighbourhood competition of understorey sapling in a subalpine forest in central Japan. Ecosci 10 (2): $217-224$

Morris EC. 2002. Self-thinning lines differ with fertility level. Ecol Res 17: $17-28$.

Morris EC. 2003. How does fertility of the substrate affect intraspecific competition? Evidence and synthesis from self-thinning. Ecol Res 18: 287-305.

Mueller-Dombois D, Ellenberg H. 1974. Aims and Methods of Vegetation Ecology. John Wiley \& Sons, New York

Murrell DJ. 2009. On the emergent spatial structure of size-structured populations: when does self-thinning lead to a reduction in clustering. J Ecol. 97: 256-266.

Nakagawa Y, Yokozawa M, Hara T. 2015. Competition among plants can lead to an increase in aggregation of smaller plants around larger ones. Ecol Mod 301: 41-53.

Newman J, Lawson S. 2005. Last Frontier Illegal Logging in Papua and China's Massive Timber Theft. Emmerson Press, Kenilworth.

Peet RK, Christensen NL, 1987. Competition and tree death. BioSci 37: 586-595.

Piao T, Chun JH, Yang HM, Cheon K. 2014. Negative density dependence regulates two tree species at later life stage in a temperate forest. Plos 9 (7): e103344.

Pierik R, Mommer L, Voesenek LACJ. 2013. Molecular mechanisms of plant competition: neighbour detection and response strategies. Funct Ecol 27: 841-853.

Potvin C, Dutilleul P. 2009. Neighborhood effects and size-asymmetric competition in a tree plantation varying in diversity. Ecol 90 (2): 321327.

Pretzsch H. 2002. Grundlage der Waldwachstumforschung. Parey-Verlag, Berlin.

Pretzsch H. 2014. Canopy space filling and tree crown morphology in mixed-species stands compared with monocultures. For Ecol Manag 327: 251-264.

Rivas JCC, Gonzalez JGA, Aguirre O, Hernandez FJ. 2005. The effect of competition on individual tree basal area growth in mature stands of Pinus cooperi Blanco in Durango (Mexico). Eur J For Res 124: $133-$ 142.

Sadono R., Sirami EV, Marsono D, Murdjoko A, Rachman I. 2014. Structural equation model for population of merbau (Intsia bijuga, Colebr. O. Kuntze) in Gunung Meja natural tourism park of Manokwari in Papua. Adv Envi Biol 8: 1012-1019.

Sakai S. 1986. Patterns of branching and extension growth of vigorous saplings of Japanese Acer species in relation to their regeneration strategies. Canadian J Bot 65: 1578-1585.

Sanchez-Salguero R, Linares JC, Camarero JJ, Madrigal-Gonzalez J, Hevia A, Sanchez-Miranda A, Ballesteros-Canovas JA, AlfaroSanchez R, Garcia-Cervigon AI, Bigler C, Rigling A. 2015. Disentangling the effects of competition and climate on individual tree growth: a retrospective and dynamic approach in scots pine. For Ecol Manag 358: 12-25.

Sansevero JBB, Prieto PV, Duarte de Moraes LF, Rodrigues PJFP. 2011 Natural regeneration in plantations of native trees in lowland Brazilian Atlantic forest: community structure, diversity, and dispersal syndromes. Rest Ecol 19 (3): 379-389.
Schwinning S, Weiner J. 1998. Mechanisms determining the degree of size asymmetry in competition among plants. Oecol 113 (4): 447455 .

Sirami EV, Marsono D, Sadono R, Imron MA. 2016. Structure, diversity, and association of liana with merbau population in Gunung Meja nature tourism park of Manokwari-West Papua. J Man Lingk 23: 8291. [Indonesian]

Sirami EV. 2014. Structure and Dynamics of Merbau (Intsia bijuga OK.) Based on Structural Equation Model in Gunung Meja Nature Tourism Park of Manokwari. [Thesis]. Gadjah Mada University, Yogyakarta. [Indonesian]

Skovsgaard JP, Vanclay JK. 2008. Forest site productivity: a review of the evolution of dendrometric concepts for even-aged stands. Forestry 81 (1): 13-31.

Statistics Agency of Manokwari Regency. 2016. Climate Situation. https://manokwarikab.bps.go.id/publikasi.html.

Stoll P, Prati D. 2001. Intraspecific aggregation alters competitive interactions in experimental plant communities. Ecology 82: 319-327.

Takenaka A. 1994. A simulation model of tree architecture development based on growth response to local light environment. J Plant Res 107: 321-330.

Teughels H, Nijs I, van Hecke P, Impens I. 1995. Competition in a global change environment: The important of different plant traits for competitive success. J Biogeograp 22 (2/3): 297-305.

Thorpe HC, Astrup R, Coates KD. 2010. Competition and tree crowns: a neighborhood analysis of three boreal tree species. For Ecol Manag 259: 1586-1596.

Tilman D. 1990. Mechanisms of plant competition for nutrients: the elements of predictive the theory of competition. In: Grace JB, Tilman D, (eds) Perspectives on Plant Competition. Academic Press, London.

Tome M, Burkhart HE. 1989. Distance-dependent competition measures for predicting growth of individual trees. For Sci 35: 816-831.

Tremmel DC, Bazzaz FA. 1993. How neighbor canopy architecture affects target plant performance. Ecology 74 (7): 2114-2124.

Tremmel DC, Bazzaz FA. 1995. Plant architecture and allocation in different neighborhoods: implications for competitive success. Ecol 76 (1): 262-271.

Tschirhart J. 2001. Resource competition among plants: from maximizing individuals to community structure. Ecol Mod 148: 191-212.

Turner IM. 2004. The Ecology of Trees in the Tropical Rain Forest. Cambridge University Press, Cambridge.

Vincent JB, Henning B, Saulei S, Sosanika G, Weiblen GD. 2015. Forest carbon in lowland Papua New Guinea: local variation and the importance of small trees. Australian Ecol 40: 151-159.

Weiner J, Berntson GM, Thomas SC. 1990. Competition and growth form in a woodland annual. J Ecol 78: 459-469.

Wickens GE, Horn HS. 1972. The adaptive geometry of trees. Kew Bull 27 (2): 377.

Wright SJ. 2002. Plant diversity in tropical forests: a review of mechanisms of species coexistence. Oecol 130: 1-14.

Zhou W, Cheng X, Wu R, Han H, Kang F, Zhu J, Tian P. 2017. Effect of intraspecific competition on biomass partitioning of Larix principisrupprechtii. Intl J Plant Biol 13 (1): 1-8.

Zhu Y, Comita LS, Hubbell SP, Ma K. 2015. Conspecific and phylogenetic density-dependent survival differs across life stages in a tropical forest. J Ecol 103: 957-966. 